\title{
Live and Heat-Killed Lactobacillus rhamnosus GG: Effects on Proinflammatory and Anti-Inflammatory Cytokines/Chemokines in Gastrostomy-Fed Infant Rats
}

\author{
NAN LI, W. MICHAEL RUSSELL, MARTHA DOUGLAS-ESCOBAR, NICK HAUSER, MARIELA LOPEZ, AND JOSEF NEU \\ Department of Pediatrics [N.L., M.D.-E., N.H., M.L., J.N.], University of Florida, Gainesville, Florida 32610; Mead-Johnson Nutrition \\ [W.M.R.], Evansville, Indiana 47721
}

\begin{abstract}
Lactobacillus rhamnosus GG (LGG), a probiotics, ameliorates intestinal and other organ inflammation in infant rats. The hypothesis is that live and heat-killed LGG have similar effects on decreasing the inflammatory response induced by E. coli lipopolysaccharide (LPS) in the infant rat. Using a gastrostomy-fed rat model, 7-d-old rat pups were gastrostomy fed with or without live LGG $\left(10^{8}\right.$ or $\left.10^{12} \mathrm{cfu} \cdot \mathrm{L}^{-1} \cdot \mathrm{kg}^{-1} \cdot \mathrm{d}^{-1}\right)$ for $6 \mathrm{~d}$. In a separate experiment, LPS was administered to rat pups with or without live or heat-killed LGG $\left(10^{8} \mathrm{cfu} \cdot \mathrm{L}^{-1} \cdot \mathrm{kg}^{-1} \cdot \mathrm{d}^{-1}\right)$. Cytokine/chemokine proteins were determined by ELISA or multiplex assay. Both live and heat-killed LGG decreased LPS-induced cytokine-induced neutrophil chemoattractant-1 (CINC-1) production in liver and plasma $(p<$ $0.05)$ and also showed a trend $(p=0.09)$ in lungs. Live and heat-killed LGG ameliorated LPS-suppressed IL-10 level in lungs $(p<0.05)$. Both forms of LGG decreased IL-1b production in liver. There was no difference between low and high doses of live LGG in the production of CINC-1, TNF- $\alpha$, and myeloperoxidase (MPO). There was a trend of increase of claudin-1 in both live and heat-killed groups $(p=0.08)$. In conclusion, both live and heat-killed LGG provided by the enteral route decrease LPS-induced proinflammatory mediators and increase anti-inflammatory mediators. (Pediatr Res 66: 203-207, 2009)
\end{abstract}

$\mathrm{T}$ he majority of microbes in the human are found in the colon and distal small intestine and consist of more than $10^{13}$ microorganisms (the "microbiota"), comprising nearly 500 species and 2 million genes (the "microbiome") $(1,2)$. This is mostly a mutually beneficial relationship, as evidenced by the important role the resident intestinal bacteria play in nutrition (3), angiogenesis (4), and mucosal immunity (5). Probiotics are live microbial agents thought to confer health benefits to the host by maintaining an advantageous intestinal microecology. One example is that probiotics may be efficacious in preventing necrotizing enterocolitis (NEC) and other diseases in infants $(6,7)$. However, caveats have been raised about their use in neonates. Case reports of sepsis from probiotics in immunocompromised infants $(8-10)$ have raised concern about short-term detrimental effects. Furthermore, establishment of the early intestinal microbiota in prematures may be long term and result in unforeseen detrimental side effects, as evidenced in recent studies (11-13).

Received January 5, 2009; accepted April 10, 2009

Correspondence: Josef Neu, M.D., Department of Pediatrics, College of Medicine, University of Florida, PO Box 100296, Gainesville, FL 32610; e-mail: neuj@peds.ufl.edu

Supported by a Grant from Mead Johnson Nutrition.
The rational for use of killed rather than live agents stems from studies showing that interactions of microbe-associated molecular patterns (MAMPs) with Toll-like receptors (TLRs) and other mucosal pattern recognition receptors (PRRs) likely mediate some of the beneficial responses of probiotics. One study reported nonvirulent Salmonella strains whose direct interaction with model human epithelia attenuate IL-8 production elicited by various proinflammatory stimuli (14). The mechanisms were found to be through abrogation of polyubiquitination of I kappa B-alpha degradation (14). More recent studies also suggest that the epithelium and resident immune cells do not simply tolerate commensal bacterial but are dependent on them (15). Commensal bacteria produce molecules such as lipopolysaccharide (LPS) (15), lipoteichoic acid (15), and CpG nucleotides (16), which in turn interact in the normal intestine with a population of surface TLRs. The resultant ongoing signaling enhances the ability of the epithelial surface to withstand injury while also priming the surface for enhanced repair responses. Therefore, either the disruption of TLR signaling or the removal of TLR ligands (derived from intestinal microorganisms) compromises the ability of the intestinal surface to protect and repair itself in the face of inflammatory or infectious insult (15). It is thus intuitive that the interactive processes of microbes with the intestine do not necessarily depend on living and thus proliferating microbes.

Our recent study in cell culture model showed that UVinactivated and live Lactobacillus rhamnosus GG (LGG) are equally effective in decreasing IL-8 production in the intestinal epithelium (17). If inactivated probiotic bacteria or their products are able to provide similar beneficial effects as the live bacteria in vivo, the experiments we propose are critical before routine use of probiotics in newborn infants. Whether live microorganisms are necessary to provide a beneficial response seen with probiotics, or whether the same killed, nonproliferating agents are able to result in similar effects requires further investigation. In this study, we hypothesized that heat killed LGG will regulate intestinal inflammation similar to live LGG.

\footnotetext{
Abbreviations: CINC-1, Cytokine-induced neutrophil chemoattractant-1, LGG, Lactobacillus rhamnosus GG, LPS, Lipopolysaccharide, MPO, myeloperoxidase, NEC, necrotizing enterocolitis
} 


\section{METHODS}

Bacteria and related preparations. LGG powder was a gift from MeadJohnson Nutritionals. Live LGG was stored at $4^{\circ} \mathrm{C}$. The activity of this powder is $\sim 3.6 \times 10^{11} \mathrm{cfu} \cdot \mathrm{g}^{-1}$. Heat-killed LGG was prepared by heating bacteria at $80^{\circ} \mathrm{C}$ for $20 \mathrm{~min}$ at a concentration of $10^{10} \mathrm{cfu} \cdot \mathrm{L}^{-1}$ in PBS and then diluted at different concentrations in rat milk substitute (RMS). Effectiveness of the heat killing method was confirmed as follows: Samples of LGG before and after heat killing were diluted in PBS and plated on L agar; $0.2 \mathrm{~mL}$ of the heat-killed sample was plated on $\mathrm{L}$ agar. The plates were incubated under anaerobic conditions for $72 \mathrm{~h}$ at $37^{\circ} \mathrm{C}$. The untreated sample was at $5.4 \times 10^{10}$ cells $\cdot \mathrm{L}^{-1}$. No colonies arose from the 0.2 -mL heat-killed sample; the minimum detection is 5000 cells $\cdot \mathrm{L}^{-1}$.

Animals. The University of Florida Institutional Animal Care and Use Committee approved the following study. In two separate experiments, Sprague-Dawley (Taconic, Germantown, NY) infant rats were randomly assigned to four gastrostomy feeding groups with five rats per group. Motherreared rats of the same age were used as reference controls. Gastrostomy feeding using the rat infant "pup-in-the-cup" model began on d 7 of life through feeding tubes constructed from $24-\mathrm{cm}$ sections of polyethylene tubing that were inserted into the stomach, as previously described (18). This is a commonly used model in studies of developmental nutrition when it is important to manipulate nutritional composition in the absence of maternal feedings. The gastrostomy placement was done under isoflurane anesthesia. Timer-controlled syringe pumps were connected to the feeding tubes and were set to feed the rats for the first $20 \mathrm{~min}$ of every hour at a weightdependent flow rate. All the gastrostomy-fed groups received the same quantity of fat and carbohydrates, and the protein component was similar to the quantity required for normal growth. Using this "pup-in-a-cup" rat model, 7 -d-old rat pups were gastrostomy fed RMS with or without LGG $\left(10^{8}\right.$ or $10^{12}$ $\mathrm{cfu} \cdot \mathrm{L}^{-1} \cdot \mathrm{kg}^{-1} \cdot \mathrm{d}^{-1}$ ) for $6 \mathrm{~d}$ to determine the appropriate dose. In a separate experiment, using the same model, LPS was administered to RMS fed rats except one group of control rats at a dose of $0.25 \mathrm{mg} \cdot \mathrm{kg}^{-1} \cdot \mathrm{d}^{-1}$ via the gastrostomy tube starting $2 \mathrm{~d}$ after the initiation of artificial feeding. This dose was determined in our pilot studies to result in occasional shivering, piloerection, and poor weight gain but was not associated with a significant increase in mortality over a 6-d period. Live or heat-killed LGG was given at dose of $10^{8} \mathrm{cfu} \cdot \mathrm{L}^{-1} \cdot \mathrm{kg}^{-1} \cdot \mathrm{d}^{-1}$.

At the end of the 6-d treatment period, the rat pups were euthanized with an overdose of pentobarbital sodium. The small intestine was removed and separated into three parts: the ileum, jejunum, and duodenum, stored at $-80^{\circ} \mathrm{C}$ for enzyme assays and ELISA, or fixed in $10 \%$ neutral-buffered formalin for intestinal morphology. Blood was obtained by cardiac puncture after euthanasia. Lung, liver, and plasma were stored at $-80^{\circ} \mathrm{C}$ for enzyme assays and ELISA.

Growth. To compare the growth rate among the dietary groups, the body weight of the pups were measured every day in the morning.

Intestinal morphology. The microscopy studies focused on the distal small intestine because this is a region that is most highly susceptible to certain pathology in VLBW infants (e.g., NEC and non-NEC-related perforations). Furthermore, pilot evaluations of the duodenum and jejunum did not demonstrate major differences in villus or crypt morphometrics. Formalin-fixed ileum samples were embedded in paraffin; $6-\mu \mathrm{m}$ sections were cut using a 2030 Reichert-Jung paraffin microtome. The sections were then stained with a routine hematoxylin and eosin (H\&E) stain. Villus height, width, and crypt depth were measured using a Nikon microscope (Universal Imaging Corp., Westchester, PA) with an ocular micrometer without the examiner knowing the group assignment. The intestinal injury was evaluated using a semiquantitative scoring system ranging from 0 to 4 modified by Arumugam et al. (19). Normal mucosa was scored as grade 0 . Epithelial cell damages, including loss of cells and separation of the epithelial cells from the underlying villus were scored between grades $1-3$, whereas loss of villus tissue was scored as grade 4 .

Myeloperoxidase assay. Intestinal total myeloperoxidase (MPO) activity, a measure of neutrophil accumulation and a marker of tissue injury, was determined by a standard enzymatic procedure as previously described (20). Briefly, intestine samples were homogenized on ice in $0.01 \mathrm{M} \mathrm{KH}_{2} \mathrm{PO}_{4}$ buffer. After centrifugation at $10,000 \times \mathrm{g}$ for $20 \mathrm{~min}$ at $4^{\circ} \mathrm{C}$, the pellets were resuspended by sonication in cetyltrimethylammonium bromide buffer (13.7 $\mathrm{mM} \mathrm{CTAB}, 50 \mathrm{mM} \mathrm{KH}_{2} \mathrm{PO}_{4}$, and $50 \mathrm{mM}$ acetic acid, $\mathrm{pH}$ 6.0). The supernatant was kept for ELISA analysis. The suspension was centrifuged again at $10,000 \times \mathrm{g}$ for $15 \mathrm{~min}$. The supernatant was then incubated in a $60^{\circ} \mathrm{C}$ water bath for $2 \mathrm{~h}$. MPO concentration of the supernatant was measured by the $\mathrm{H}_{2} \mathrm{O}_{2}$-dependent oxidation of tetramethylbenzidine. Absorbance was determined at $650 \mathrm{~nm}$ and compared with a linear standard curve. Protein was measured using the BioRad Dc Protein Assay.

Reagents and cytokine multiplex assays. To screen for additional proinflammatory and anti-inflammatory mediators, multiplex assays were per- formed. Multiplex bead kits were purchased from LINCO Research, Inc. (St. Charles, MO). Cytokines/chemokines were analyzed by a kit that included the following: granulocyte-macrophage colony-stimulating factor (GMCSF), IFN- $\gamma$, IL-1 $\alpha$, IL-1 $\beta$, IL-2, IL-4, IL-5, IL-6, IL-10, IL-12p70, IL-18, monocyte chemoattractant protein-1 (MCP-1), GRO/KC [rat cytokine-induced neutrophil chemoattractant-1 (CINC-1), rodent IL-8 equivalent chemokine], and TNF- $\alpha$. Multiplex assay was performed according to the manufacturer's instructions. Standard curves for each cytokine/chemokine were generated by using the reference concentrations supplied by the manufacturers. Raw data (mean fluorescent intensity) were analyzed by MasterPlex Quantitation Software (MiraiBio, Inc., Alameda, CA) to obtain concentration values.

ELISA for CINC-1, TNF- $\alpha$, and $\boldsymbol{I L}-10$. After screened by Cytokine Multiplex Assays, certain cytokines were evaluated by ELISA. Samples were isolated from cellular extracts of whole tissues. To evaluate proinflammatory and anti-inflammatory cytokines, liver, lungs, small intestine, and plasma CINC-1, IL-10, and TNF- $\alpha$ were determined by TiteZyme Enzyme Immunometric Assay kits for rat growth-related oncogene (GRO)/CINC-1, IL-10, and TNF- $\alpha$ (Assay Designs, Ann Arbor, MI), respectively. Absorbance was determined at $450 \mathrm{~nm}$, and concentration was calculated using the equation derived from a linear standard curve.

Immunoblotting for tight junctions. Small intestinal samples were harvested and homogenized in western lysis buffer $(10 \mathrm{mM}$ Tris-HCl, $\mathrm{pH} 7.6$, $150 \mathrm{mM} \mathrm{NaCl}, 2 \mathrm{mM}$ EDTA, 1\% SDS) with protease inhibitors. Protein was measured using the BioRad Dc Protein Assay (BioRad, Hercules, CA). Equal amount of protein from each sample was separated on $12.5 \%$ SDS-PAGE and then transferred to PVDF membrane. Blots were blocked by $5 \%$ nonfat milk and incubated with primary antibodies (claudin-1, occludin obtained from Zymed Laboratory Inc., South San Francisco, CA) at $4^{\circ} \mathrm{C}$ overnight. Membranes were incubated sequentially with horseradish peroxidase-conjugated secondary antibody and detected with ECL plus (Amersham Pharmacia Biotech, Piscataway, NJ).

Statistical analysis. SigmaStat statistical software (SPSS, Chicago, IL) was used to analyze body weight, MPO, ELISA for CINC-1, TNF- $\alpha$, and cytokine/chemokine multiplex assay results. All data were reported as mean \pm SD. A one-way ANOVA was used to determine whether a significant difference was present among the treatment groups. The Holm-Sidak method was performed for pairwise comparisons when the ANOVA was significant at $p<0.05$.

\section{RESULTS}

Animal weights. There was no difference in mortality among the groups. Rat pups were weighed daily after the gastrostomy and compared with mother-fed reference animals. The motherfed animals grew more rapidly than the LPS-treated, gastrostomy fed pups (data not shown). Providing live or heat-killed LGG probiotics to gastrostomy-fed, LPS-treated pups did not improve weight gain.

CINC-1 peptide from intestine, liver, plasma, and lung. ELISA results showed that LPS increased CINC-1 levels in liver (Fig. 1A), lungs (Fig. 1B), and plasma (Fig. 1C). Both live and heat-killed LGG decreased LPS-induced CINC-1 production in liver (Fig. 1A) and plasma (Fig. $1 C ; p<0.05$, $n=4-7)$ and also showed a trend $(p=0.09, n=4-7)$ in lungs (Fig. 1B). No significant difference was seen in intestine (data not shown). Cytokine multiplex assay showed similar results for CINC-1 (GRO/KC).

$I L-1 \alpha, I L-1 \beta, I L-2, I L-12, I L-18$, and MCP-1 peptide from intestine, liver, plasma, and lung. Cytokine multiplex assay showed that both live and heat-killed LGG decreased IL-1 $\beta$ production in liver $(p<0.05)$ but not in lungs (Fig. 2$)$. No significant difference was seen with IL- $1 \alpha$, IL-2, IL-12, IL-18, and MCP-1 in various tissues (data not shown).

IL-10 peptide from liver, lung, and plasma. ELISA showed that LPS decreased anti-inflammatory cytokine IL-10 in lungs (Fig. 3), but caused no change in liver and plasma (data not shown). Both live and heat-killed-LGG increased LPSsuppressed IL-10 level in lungs (Fig. 3). 

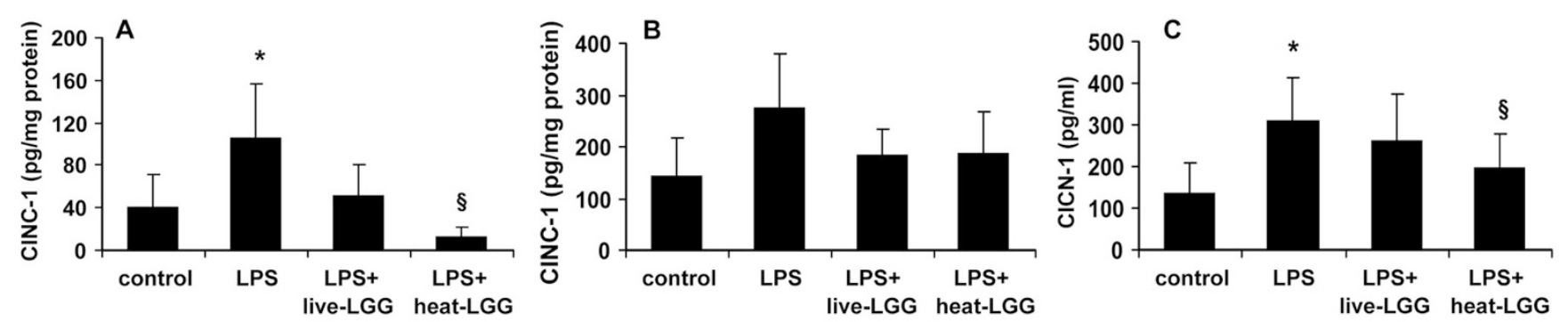

Figure 1. Effects of live and heat-killed LGG on LPS-induced CINC-1. ELISA results showed that both live and heat-killed LGG decreased LPS-induced CINC-1 production in liver $(A, p<0.01)$ and plasma $(C, p<0.05)$ and also showed a trend $(p=0.09)$ in lungs $(B)$. All data were reported as mean \pm SD. $n=4-7 . * p<0.05 v s$ control; $\S p<0.05 v s$ LPS.

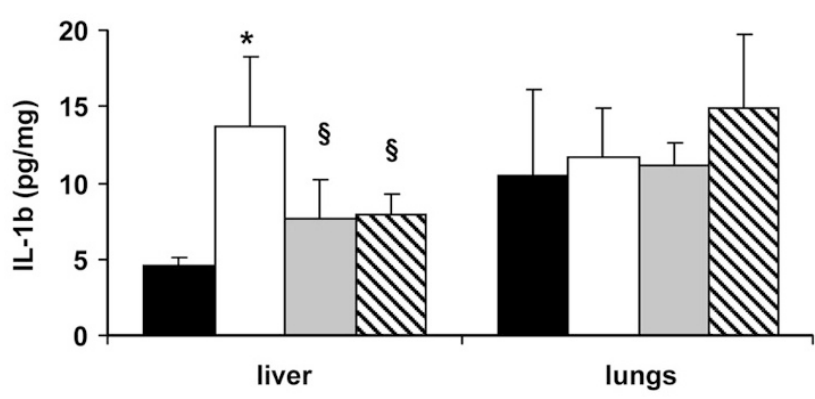

Figure 2. Effects of live and heat-killed LGG on LPS induced IL-1 $\beta$ Peptide. Cytokine multiplex assay showed that both live and heat-killed LGG decreased IL-1 $\beta$ production in liver $(p<0.05)$ but not in lungs. All data were reported as mean \pm SD. $n=4-7$. $* p<0.05 v s$ control; $\$ p<0.05 v s$ LPS. $\square$, control; $\square$, LPS; $\square$, live-LGG; $\mathbf{Q}$, heat-LGG.

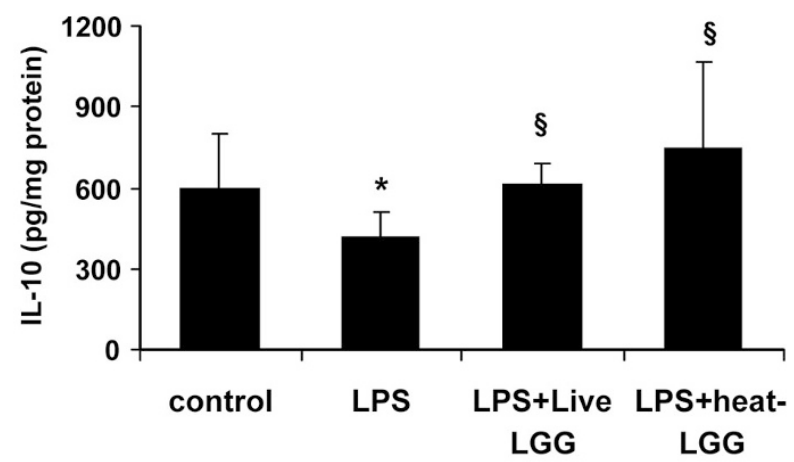

Figure 3. Effects of live and heat-killed LGG on IL-10 peptide. ELISA showed that LPS decreased anti-inflammatory cytokine IL-10 in lungs $(p<$ $0.05)$. Live and heat-killed-LGG increased LPS-suppressed IL-10 level in lungs $(p<0.05)$. All data were reported as mean \pm SD. $n=4-7 . * p<0.05$ vs control; $\$ p<0.05$ vs LPS.

TNF- $\alpha$ peptide from plasma and lung. There was no difference in TNF- $\alpha$ level in liver, intestine, and lungs among the groups by both cytokine multiplex assay and ELISA (data not shown).

Intestinal histology. To compare intestinal morphology among the dietary groups, small intestinal villus morphology was evaluated using light microscopy (Fig. 4). Three animals from each group were evaluated. The distal small intestinal villus lengths, widths, and crypt depths did not differ significantly within the gastrostomy-fed groups (data not shown). Representative sections of ileum from LPS-treated rat pups showed a greater distortion of overall villus architecture (Fig. $4 B)$ and with higher injury score $(p<0.05$; Fig. $4 E)$ than the other groups. Live (Fig. 4C) and heat-killed LGG (Fig. 4D) groups were more similar to the control group (Fig. 4A).

Low-dose $L G G$ versus high-dose $L G G$. ELISA results showed that both the low $\left(10^{8} \mathrm{cfu} \cdot \mathrm{L}^{-1} \cdot \mathrm{kg}^{-1} \cdot \mathrm{d}^{-1}\right)$ and high dose $\left(10^{12} \mathrm{cfu} \cdot \mathrm{L}^{-1} \mathrm{~kg}^{-1} \cdot \mathrm{d}^{-1}\right)$ of live LGG decreased TNF- $\alpha$ in liver (Fig. $5 A ; p<0.05$ ) and MPO in lungs (Fig. $5 B$; $p<0.05)$. However, no difference was seen between low- and high-dose groups in TNF- $\alpha$ (Fig. 5A) and MPO (Fig. 5B) levels. There is no difference between low- and high-dose of live LGG in the production of CINC-1 in various tissues (data not shown). These results suggest that the administration of LGG at the higher dose results in similar down-regulation of inflammatory mediators as the low dose.

Tight junction proteins claudin-1 and occludin. Western blot showed that LPS did not alter the expression of both claudin-1 and occludin expression (data not shown). There was a trend of increase of claudin- 1 in both live and heatkilled groups $(p=0.08)$ by $20 \%$ and $59 \%$, respectively, compared with LPS group. No significant difference was seen among the four groups in occludin (data not shown).

\section{DISCUSSION}

The results of these experiments demonstrated that both live and heat-killed LGG provided by the enteral route diminishes LPS-induced proinflammatory mediators and up-regulate antiinflammatory mediators in liver, plasma, and lung, suggesting that the killed microbe has similar effects to that of the live microbe.

Numerous benefits have been shown for probiotics in promotion of health and prevention of disease. Evidence is accumulating that the interaction of the luminal microbes with the cells of the intestinal epithelium and how they communicate through both innate and adaptive immune systems plays a significant role in subsequent health, including autoimmune diseases, allergies, asthma, and gastrointestinal illnesses (21). Probiotics have been shown to decrease the duration and severity of rotavirus-related diarrhea, allergies to cow-milk proteins, atopic dermatitis, and some inflammatory diseases of the GI tract (22).

Neonatologists are anxiously poised for the routine administration of probiotics to premature infants even without a prior systematic analysis of its long-term safety. Recent sys- 


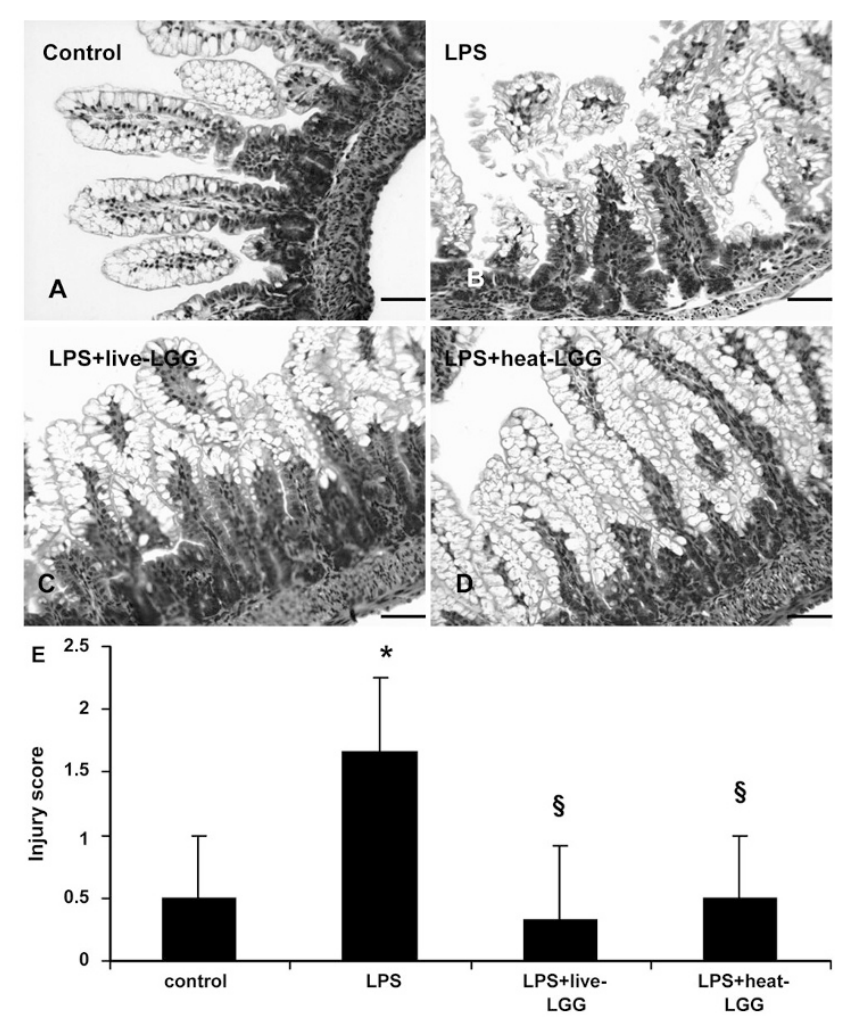

Figure 4. Effects of live and heat-killed LGG on intestinal histology. Small intestinal villus morphology was evaluated using light microscopy. Representative sections of ileum from LPS-treated rat pups showed a greater distortion of overall villus architecture $(B)$ and than the other groups. Live $(C)$ and heat-killed LGG $(D)$ groups are more similar to the control group $(A)$. E, The intestinal injury was evaluated using a semiquantitative scoring system ranging from 0 to 4 . Normal mucosa was scored as grade 0 . Epithelial cell damage, including loss of cells and separation of the epithelial cells from the underlying villus were scored between grades 1 and 3 , whereas loss of villus tissue was scored as grade 4. $n=3$. Scale bar $=25 \mu \mathrm{m}$; magnification was $\times 250 . * p<0.05 v s$ control; $\$ p<0.05 v s$ LPS.

tematic reviews (23-26) tout the potential of probiotics in the prevention of NEC in premature infants. Along with enthusiasm, caution is being expressed in that the scientific basis as well as short- and long-term safety have not been established $(27,28)$. In a multicenter double-blind study from Italy (29), preterm infants were randomized to receive either placebo or LGG, and the incidence of urinary tract infection, bacterial sepsis, and NEC were examined. Although there seemed to be a decrease in NEC in treated infants, this reduction was not statistically significant. In an open study from Bogota, Colombia, Hoyos (30) reported a reduction in the incidence of NEC in infants in a neonatal intensive care unit after the prophylactic administration of probiotic supplemented enteral feeding. The comparison was made with historical controls, the treating physicians were not blinded, and the study subjects generally had higher birth weights and were more mature. Nevertheless, they reported an almost 3-fold decrease in cases of NEC and a 4-fold decrease in NEC-related mortality. Two prospective, randomized blinded studies $(6,7)$ reported a decreases in NEC and NEC plus mortality following probiotic prophylaxis.

Despite the recent enthusiasm for probiotic usage, there were cautionary warnings about indiscriminant manipulation
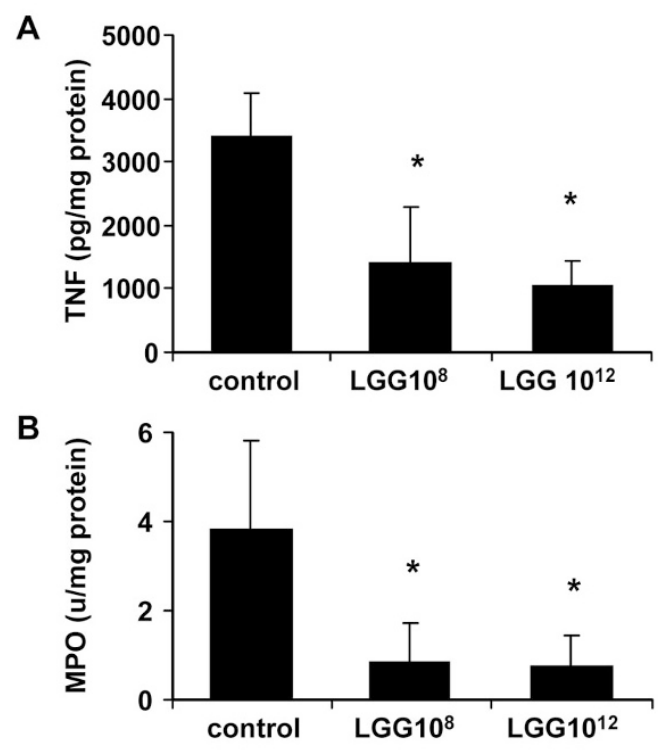

Figure 5. Low-dose LGG $v s$ high-dose LGG. ELISA results showed that both low dose $\left(10^{8} \mathrm{cfu} \cdot \mathrm{L}^{-1} \cdot \mathrm{kg}^{-1} \cdot \mathrm{d}^{-1}\right)$ and high dose $\left(10^{12} \mathrm{cfu} \cdot \mathrm{L}^{-1} \mathrm{~kg}^{-1} \cdot \mathrm{d}^{-1}\right)$ of live LGG decreased TNF- $\alpha$ in liver $(A, p<0.05)$ and MPO in lungs $(B, p<$ $0.05)$. No difference was seen between low-dose and high-dose group. All data were reported as mean \pm SD. $n=4-7$. ${ }^{*} p<0.05$ vs control.

of the GI microbiota during the perinatal and neonatal time periods and in individuals who may be immunosuppressed $(27,28)$. The ramifications of early probiotic usage may be long term. Studies using nonculture-based comprehensive analyses have just recently been initiated (31). Similarly, studies of long-term health outcomes have just begun and are raising concerns. One 7-y follow-up study (11) where LGG was administered to mothers before delivery and then to the infants shortly after delivery showed decreased atopic dermatitis in the group receiving LGG. However, there were more cases of allergic rhinitis and asthma in the Lactobacillus GG group at $7 \mathrm{y}$ of age. Although not statistically significant, the strong trend raises concern that attention needs to be given to long-term health effects of early probiotic administration. More recent studies amplify the need for concern. One study from Germany observed neither a preventive effect of LGG on the development of atopic dermatitis nor any trend in this direction with LGG, but there was a significant increase in wheezing bronchitis in the LGG-treated group (12). Another study from Australia, points in the same direction regarding allergic sensitization, also suggesting that the routine use of probiotics for prevention of illness must be exercised with caution (13). At the age of $12 \mathrm{mo}$, the rate of sensitization to common allergens was significantly higher in the probiotic group (13).

The concerns being raised to the use of live agents in the immediate neonatal period as well as for the use in premature infants has prompted the need for studies of agents that may not remain in the intestine long term. Prebiotics, although of potential benefit, may still promote the growth of whatever organisms are most prevalent in the environment, which may not be optimal when infants are housed in the neonatal intensive care unit, where pathogenic microorganisms may be prevalent. Thus, use of killed probiotics that possess similar 
MAMPs to those of the live microbes, but that will not proliferate long term may provide added safety as long as efficacy can be established.

Previous studies have demonstrated that addition of heatkilled L. acidophilus LB to oral rehydration therapy was effective in the treatment of children with acute diarrhea by decreasing the duration of diarrhea (32). Others have shown that heat-killed L. acidophilus LB plus its culture medium antagonizes the C1845-induced increase in paracellular permeability in intestinal Caco-2/TC7 cells and produces a clinically significant benefit in the management of children with nonrotavirus diarrhea (33). Our study shows that LPS-induced inflammation can be modulated by the use of both live and heat killed LGG. Of interest is that the intestinal mucosa did not show a significant effect on proinflammatory mediator production with either LPS or the live or heat-killed LGG, but an effect was seen in the plasma and distal organs such as the liver and lung. The differences in the intestine from our previous experiments where there was an effect of LPS and the live probiotic on the intestinal mucosa may relate to dosage used in the different experiments or may also be due to interexperimental differences. Nevertheless, significant differences were noted in the plasma, liver, and lung with both cytokine multiplexing methodology and ELISA.

We interpret these results as showing that dead microbes may be as effective as live agents in modulation of inflammation. Other studies have suggested that $\mathrm{CpG}$ (16) or even TLR agonists such as LPS and lipoteichoic acid (15) in low doses may be effective in promoting the inhibitory actions of the intestinal epithelium in the signaling cascade of the inflammatory response (34). The biologic basis for the heat-killed LGG might be the cell components, such as cell wall, LTA, and peptidoglycan. These cell components would serve as TLRs agonists to induce the tolerance to a later pro-inflammatory stimulus, such as LPS. Further work on specific mechanisms is needed to delineate what is actually being done by the dead agents and whether this is similar to the mechanisms of the live agents.

Intestinal barrier function may also play an important role in preventing the bacterial translocation and bacteremia. Although our study did not show the significant changes of tight junction protein occludin and claudin-1, further studies need to be done to investigate the effects of probiotics on the barrier function, including other tight junction proteins, such as ZO-1, claudins $2,3,4,5$, and 7 .

In summary, we show for the first time in an animal model that heat-killed LGG has similar effects in modulating splanchnic-derived inflammation that can be reflected in plasma and a distal organ such as the lung. This may be a safer approach than using live microbes in certain situations such as immediately after birth, in premature infants, or in immunosuppressed individuals.

\section{REFERENCES}

1. Xu J, Gordon JI 2003 Honor thy symbionts. Proc Natl Acad Sci USA 100:1045210459

2. Hooper LV, Midtvedt T, Gordon JI 2002 How host-microbial interactions shape the nutrient environment of the mammalian intestine. Annu Rev Nutr 22:283-307

3. Kien CL 1996 Digestion, absorption, and fermentation of carbohydrates in the newborn. Clin Perinatol 23:211-228
4. Hooper LV, Stappenbeck TS, Hong CV, Gordon J 2003 Angiogenins: a new class of microbicidal proteins involved in innate immunity. Nat Immunol 4:269-273

5. Bourlioux P, Koletzko B, Guarner F, Braesco V 2003 The intestine and its microflora are partners for the protection of the host: report on the Danone Symposium "The Intelligent Intestine," held in Paris, June 14, 2002. Am J Clin Nutr 78:675-683

6. Bin-Nun A, Bromiker R, Wilschanski M, Kaplan M, Rudensky B, Caplan M, Hammerman C 2005 Oral probiotics prevent necrotizing enterocolitis in very low birth weight neonates. J Pediatr 147:192-196

7. Lin HC, Su BH, Chen AC, Lin TW, Tsai CH, Yeh TF, Oh W 2005 Oral probiotics reduce the incidence and severity of necrotizing enterocolitis in very low birth weight infants. Pediatrics 115:1-4

8. Berger RE 2005 Lactobacillus sepsis associated with probiotic therapy. J Urol $174: 1843$

9. Dhaliwal R, Heyland DK 2005 Nutrition and infection in the intensive care unit: what does the evidence show? Curr Opin Crit Care 11:461-467

10. De Groote MA, Frank DN, Dowell E, Glode MP, Pace NR 2005 Lactobacillus rhamnosus GG bacteremia associated with probiotic use in a child with short gut syndrome. Pediatr Infect Dis J 24:278-280

11. Kalliomaki M, Salminen S, Poussa T, Isolauri E 2007 Probiotics during the first 7 years of life: a cumulative risk reduction of eczema in a randomized, placebocontrolled trial. J Allergy Clin Immunol 119:1019-1021

12. Kopp MV, Hennemuth I, Heinzmann A, Urbanek R 2008 Randomized, doubleblind, placebo-controlled trial of probiotics for primary prevention: no clinical effects of Lactobacillus GG supplementation. Pediatrics 121:e850-e856

13. Taylor AL, Dunstan JA, Prescott SL 2007 Probiotic supplementation for the first 6 months of life fails to reduce the risk of atopic dermatitis and increases the risk of allergen sensitization in high-risk children: a randomized controlled trial. J Allergy Clin Immunol 119:184-191

14. Neish AS, Gewirtz AT, Zeng H, Young AN, Hobert ME, Karmali V, Rao AS, Madara JL 2000 Prokaryotic regulation of epithelial responses by inhibition of IkappaB-alpha ubiquitination. Science 289:1560-1563

15. Rakoff-Nahoum S, Paglino J, Eslami-Varzaneh F, Edberg S, Medzhitov R 2004 Recognition of commensal microflora by toll-like receptors is required for intestinal homeostasis. Cell 118:229-241

16. Lee J, Rachmilewitz D, Raz E 2006 Homeostatic effects of TLR9 signaling in experimental colitis. Ann N Y Acad Sci 1072:351-355

17. Lopez M, Li N, Kataria J, Russell M, Neu J 2008 Live and ultraviolet-inactivated Lactobacillus rhamnosus GG decrease flagellin-induced interleukin-8 production in Caco-2 cells. J Nutr 138:2264-2268

18. Hall WG 1975 Weaning and growth of artificially-reared rats. Science 190:13131315

19. Arumugam TV, Arnold N, Proctor LM, Newman M, Reid RC, Hansford KA, Fairlie DP, Shiels IA, Taylor SM 2003 Comparative protection against rat intestinal reperfusion injury by a new inhibitor of sPLA2, COX-1 and COX-2 selective inhibitors, and an LTC4 receptor antagonist. Br J Pharmacol 140:71-80

20. Auricchio R, Paparo F, Maglio M, Franzese A, Lombardi F, Valerio G, Nardone G, Percopo S, Greco L, Troncone R 2004 In vitro-deranged intestinal immune response to gliadin in type 1 diabetes. Diabetes 53:1680-1683

21. Bach JF 2002 The effect of infections on susceptibility to autoimmune and allergic diseases. N Engl J Med 347:911-920

22. Vanderhoof JA, Young RJ 2001 The role of probiotics in the treatment of intestinal infections and inflammation. Curr Opin Gastroenterol 17:58-62

23. Alfaleh K, Bassler D 2008 Probiotics for prevention of necrotizing enterocolitis in preterm infants. Cochrane Database Syst Rev CD005496

24. Barclay AR, Stenson B, Simpson JH, Weaver LT, Wilson DC 2007 Probiotics for necrotizing enterocolitis: a systematic review. J Pediatr Gastroenterol Nutr 45:569-576

25. Deshpande G, Rao S, Patole S 2007 Probiotics for prevention of necrotising enterocolitis in preterm neonates with very low birthweight: a systematic review of randomised controlled trials. Lancet 369:1614-1620

26. Martin CR, Walker WA 2008 Probiotics: role in pathophysiology and prevention in necrotizing enterocolitis. Semin Perinatol 32:127-137

27. Boyle RJ, Robins-Browne RM, Tang ML 2006 Probiotic use in clinical practice: what are the risks? Am J Clin Nutr 83:1256-1264

28. Neu J 2007 Perinatal and neonatal manipulation of the intestinal microbiome: a note of caution. Nutr Rev 65:282-285

29. Dani C, Biadaioli R, Bertini G, Martelli E, Rubaltelli FF 2002 Probiotics feeding in prevention of urinary tract infection, bacterial sepsis and necrotizing enterocolitis in preterm infants. A prospective double-blind study. Biol Neonate 82:103-108

30. Hoyos AB 1999 Reduced incidence of necrotizing enterocolitis associated with enteral administration of Lactobacillus acidophilus and Bifidobacterium infantis to neonates in an intensive care unit. Int J Infect Dis 3:197-202

31. Palmer C, Bik EM, Digiulio DB, Relman DA, Brown PO 2007 Development of the Human Infant Intestinal Microbiota. PLoS Biol 5:e177

32. Simakachorn N, Pichaipat V, Rithipornpaisarn P, Kongkaew C, Tongpradit P, Varavithya W 2000 Clinical evaluation of the addition of lyophilized, heat-killed Lactobacillus acidophilus LB to oral rehydration therapy in the treatment of acute diarrhea in children. J Pediatr Gastroenterol Nutr 30:68-72

33. Liévin-Le Moal V, Sarrazin-Davila LE, Servin AL 2007 An experimental study and a randomized, double-blind, placebo-controlled clinical trial to evaluate the antisecretory activity of Lactobacillus acidophilus strain LB against nonrotavirus diarrhea. Pediatrics 120:e795-e803

34. Shibolet O, Podolsky DK 2007 TLRs in the Gut. IV. Negative regulation of Toll-like receptors and intestinal homeostasis: addition by subtraction. Am J Physiol Gastrointest Liver Physiol 292:G1469-G1473 